\title{
Bioengineered Acellular Vessel Implantation in a Patient with Chronic Limb-Threatening Ischemia: A Case Report and Discussion of Implications for Trauma
}

\author{
Alexis L Lauria, Joseph M White, Alexander J Kersey, Paul W White \\ and Todd E Rasmussen
}

Department of Surgery, Uniformed Services University of the Health Sciences and Walter Reed National Military Medical Center, Bethesda, Maryland, USA

Keywords: Bioengineered Blood Vessel; Vascular Reconstruction; Vascular Trauma; Novel Conduit

\section{BACKGROUND}

While the field of vascular surgery has seen major advances over the past few decades, options for vascular conduits in open bypass reconstruction remain largely unchanged. Current available conduits include autologous vein, synthetic grafts, and cadaveric and xenogeneic tissues. Autologous vein is frequently limited by availability, and vein harvest adds significant operative time to the procedure. Synthetic grafts suffer from an increased risk of infection, limiting use in an infected or contaminated field. Additionally, synthetic, cadaveric, and xenogeneic grafts have inferior patency when compared to autologous conduit [1]. The Human Acellular Vessel (HAV)

\section{Corresponding author:}

Alexis Lauria, Walter Reed National Military Medical Center, 8901 Rockville Pike, Bldg 9A, Ste 2122, Bethesda, MD 20889, USA.

Email: alexis.I.lauria.mil@mail.mil

Disclosure: The opinions or assertions contained herein are the private ones of the author/speaker and are not to be construed as official or reflecting the views of the Department of Defense, the Uniformed Services University of the Health Sciences or any other agency of the U.S. Government. The authors do not have financial interests in this Humacyte product, nor are they endorsing its use.

(C) 2021 CC BY 4.0 - in cooperation with Depts. of Cardiothoracic/ Vascular Surgery, General Surgery and Anesthesia, Örebro University Hospital and Örebro University, Sweden
(Humacyte, Inc., Durham, NC) is a bioengineered blood vessel that can be obtained "off the shelf" and has shown promise in the areas of durability, tissue incorporation, and resistance to infection [2-4]. A conduit with these properties carries substantial potential for use across the spectrum of vascular disease. We describe a case in which the HAV was utilized for revascularization in a patient with chronic, limb-threatening ischemia (CLTI) who lacked alterative reconstructive options. The case is followed by a brief discussion of potential broader applications of the HAV, specifically for use in vascular trauma.

\section{CASE PRESENTATION}

A 70-year-old male with a complex vascular history to include previous endovascular repair of an infrarenal abdominal aortic aneurysm and peripheral arterial disease (PAD) presented with CLTI of the right lower extremity. Examination was notable for non-palpable popliteal and pedal pulses with a monophasic dorsalis pedis artery (DPA) signal, and an ankle-brachial index (ABI) of 0.1. Arterial duplex ultrasound (DUS) demonstrated patent right superficial femoral artery (SFA), totally occluded right popliteal artery at Hunter's canal, and lack of flow through the anterior tibial, peroneal, and posterior tibial arteries. Diagnostic angiography was completed and demonstrated chronic total occlusion of the above knee popliteal artery with proximal reconstitution of the peroneal artery and distal reconstitution of the 

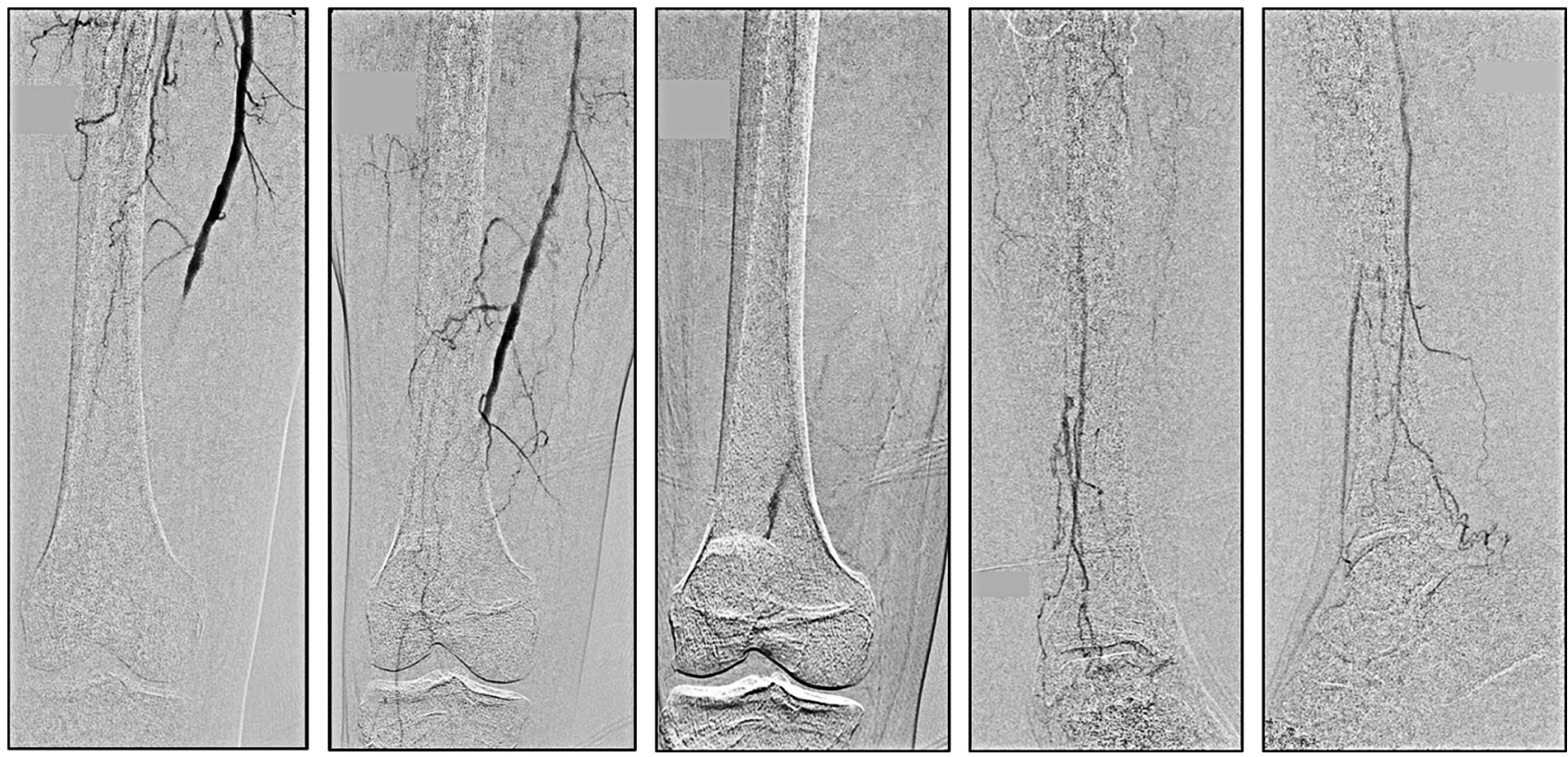

Figure 1 Preoperative angiography demonstrating total occlusion of the above knee popliteal artery with distal reconstitution of the peroneal and anterior tibial arteries.
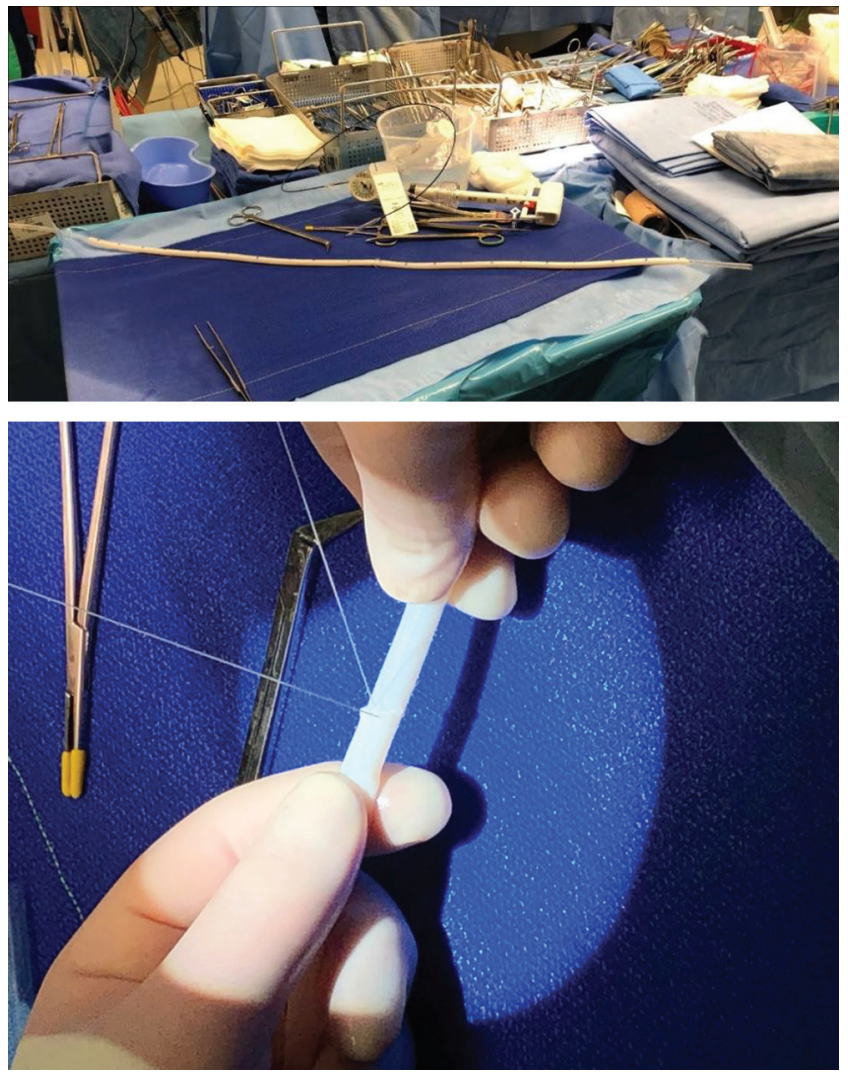

Figure 2 Intraoperative images of the human acellular vessel (HAV).

anterior tibial artery and dorsalis pedis artery (Figure 1). The lesion was not amendable to endovascular treatment.

Vein mapping was performed and showed no suitable autologous vein for conduit. Compassionate use of the

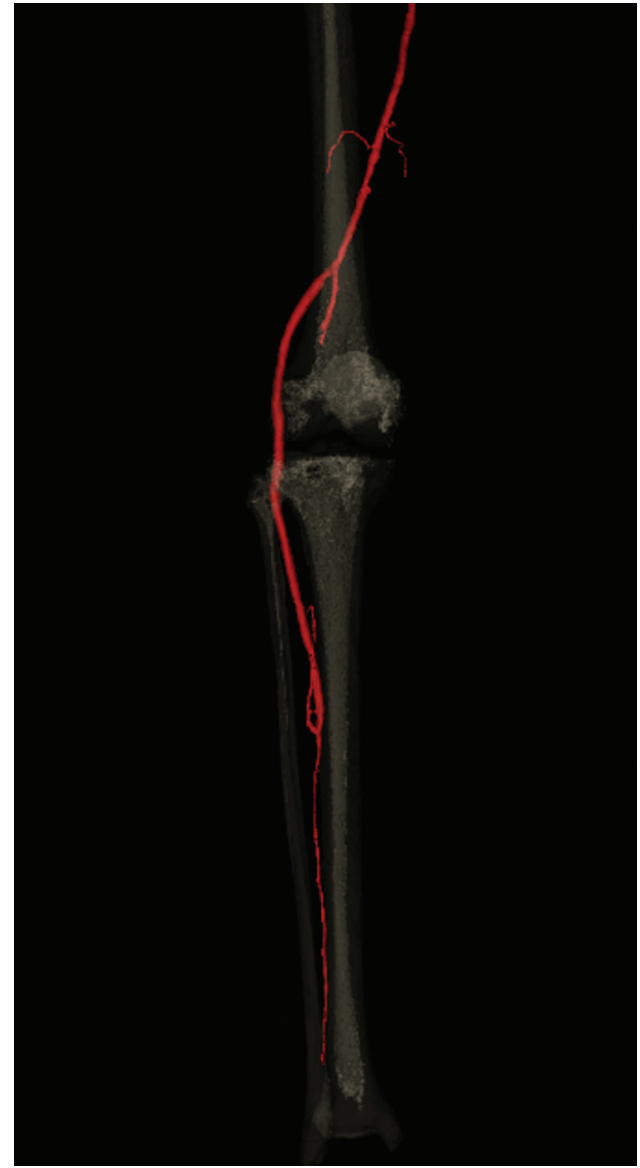

Figure 3 Three-dimensional reconstruction of right lower extremity computed tomography angiography (CTA) at 9 months postoperatively, showing patent right distal superficial femoral artery to proximal peroneal artery bypass with HAV. 
HAV was requested via the U.S. Food and Drug Administration (FDA) Expanded Access Pathway, and the patient underwent an uncomplicated distal SFA to proximal peroneal artery bypass with a single $6 \mathrm{~mm} \times 40 \mathrm{~cm}$ HAV implant creating an approximately $30 \mathrm{~cm}$ bypass (Figure 2). Anastomoses were performed with Gore-Tex ${ }^{\mathrm{TM}}$ (Gore, Newark, DE) suture, and the implant was tunneled anatomically. Postoperatively, the patient did well with return of a distal pulse and resolution of his rest pain. A right lower extremity computed tomography angiography (CTA) at 9 months (Figure 3), angiogram at 11 months (Figure 4), and DUS at 20 months postoperatively demonstrated primary patency of the implant. The patient has not sustained an infectious complication or immune rejection of the HAV implant. With the exception of chronic right lower extremity neuropathy and paresthesia, which had been present prior to the reconstruction and remain stable, the patient has continued to do well and is now over 22 months from surgery.

\section{Ethical Approval and Informed Consent}

Ethical approval and informed consent were not required for this retrospective case report. All information has been anonymised.

\section{DISCUSSION}

We describe a case in which the HAV was implanted for reconstruction in CLTI. The HAV has been studied in above-knee reconstruction for PAD [2]; however, to the authors' knowledge this is the first report of a successful bypass to a tibial artery using HAV. FDA emergency authorization for compassionate use allowed timely, effective restoration of blood flow to the patient's limb, and nearly two years later the patient has continued flow through the implant.

Intraoperative handling of the HAV was favorable. HAV mimics the properties of autologous vein more closely than those of synthetic conduits. Tunneling and creation of anastomoses using standard techniques was uncomplicated. No unanticipated technical challenges were encountered during surgical implantation.

While the indication for HAV implantation in this patient was CLTI, utility of the conduit may extend to multiple disease processes within vascular surgery. In particular, an "off-the-shelf", infection-resistant conduit is of significance to military vascular trauma. Epidemiologic analyses of injuries sustained in Operation Enduring Freedom (OEF) and Operation Iraqi Freedom (OIF) have demonstrated rates of vascular injury of up
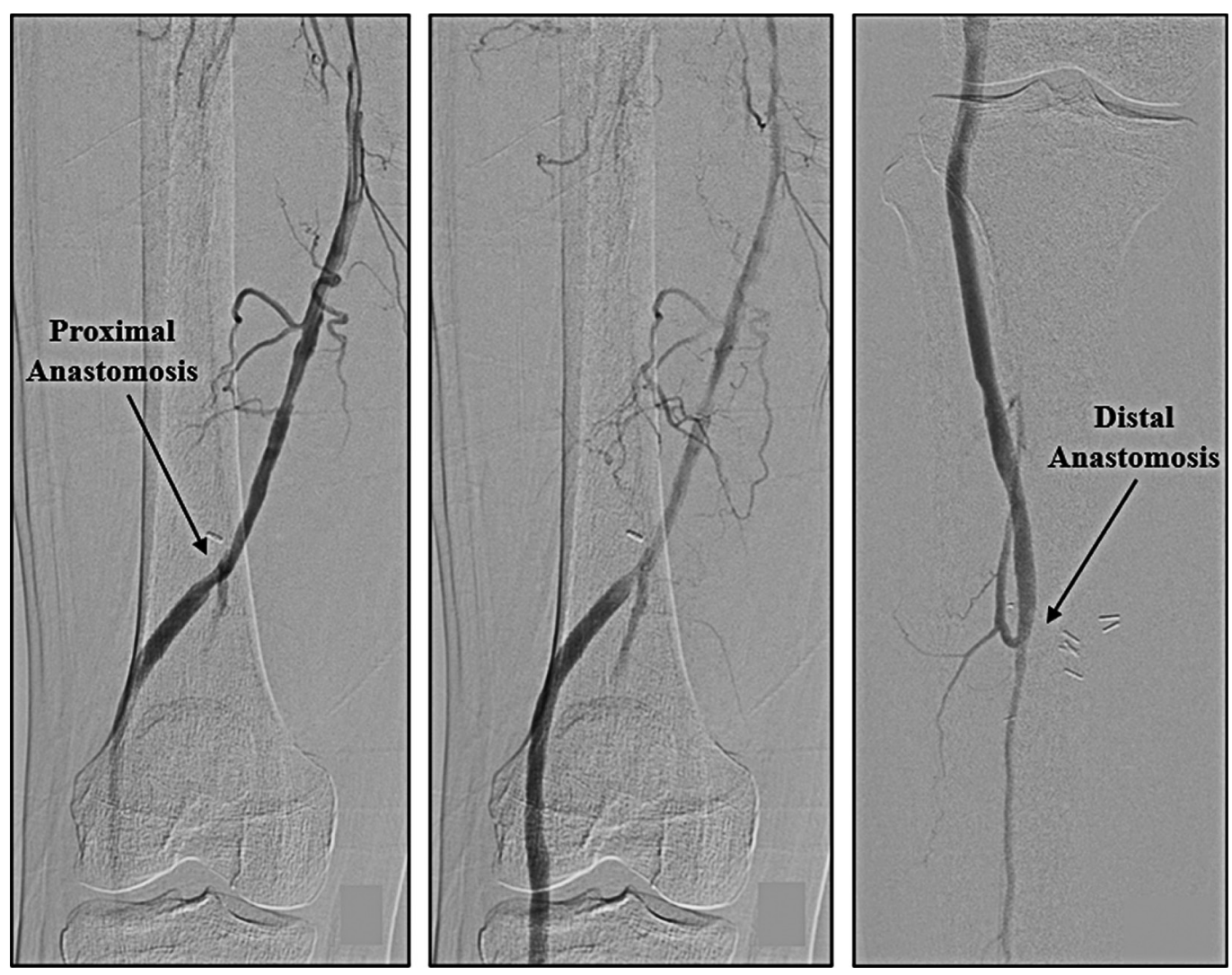

Figure 4 Right lower extremity angiogram performed at 11 months postoperatively demonstrating widely patent bypass. 
to $18 \%$, which is nearly five times that reported in previous wars, with extremity vascular injuries being most common (70-80\%). Additionally, reconstructive procedures are performed more frequently, in up to $57 \%$ of cases, with the highest rates of reconstruction seen in the second half of the Global War on Terrorism (GWOT) [5-7].

The Joint Trauma System Clinical Practice Guidelines (JTS CPG) recommend autologous vein for reconstruction of injured extremity arteries, with prosthetic graft as a last resort and preferably only in wounds with minimal contamination [8]. However, in combat-associated wounds, significant contamination frequently occurs amidst complex explosive mechanisms with secondary blast injury. These injury patterns limit conduit options as autologous vein may be damaged or, if available, time required to harvest vein may preclude its use in soldiers in critical condition with multiple concomitant injuries [1]. While prosthetic grafts offer availability and sizing options, and may allow for short-term revascularization, the risk of thrombosis or subsequent infection requiring explanation remains high [9]. Additionally, high rates of graft rupture with subsequent life-threatening hemorrhage have been reported with vein grafts in infected wounds [10]. A conduit with long-term durability that can be expediently placed for extremity revascularization in extensive, contaminated wounds would be a step forward for successful limb salvage in the young, military population.

The HAV, an "off-the-shelf", infection-resistant conduit may be limb-saving in cases where autologous vein or prosthetic use is limited. Continued real-world experience with the HAV and ongoing clinical trials [11] may lead to significant improvement in the management of vascular disease and trauma.

\section{Ethics Statement}

(1) All the authors mentioned in the manuscript have agreed to authorship, read and approved the manuscript, and given consent for submission and subsequent publication of the manuscript.

(2) The authors declare that they have read and abided by the JEVTM statement of ethical standards including rules of informed consent and ethical committee approval as stated in the article.

\section{Conflicts of Interest}

The authors declare that they have no conflicts of interest.

\section{Funding}

The authors received no financial support for the research, authorship, and/or publication of this article.

\section{Author Contributions}

TER and JMW were responsible for conception and design. ALL carried out the data collection and article drafting. TER, JMW, PWW, ALL and AJK performed the critical revisions.

\section{REFERENCES}

[1] Morrison JJ, McMahon J, DuBose JJ, Scalea TM, Lawson JH, Rasmussen TE. Clinical implementation of the Humacyte human acellular vessel: implications for military and civilian trauma care. J Trauma Acute Care Surg. 2019;87(1S Suppl 1):S44-7.

[2] Gutowski P, Gage SM, Guziewicz M, et al. Arterial reconstruction with human bioengineered acellular blood vessels in patients with peripheral arterial disease. J Vasc Surg. 2020;72(4):1247-58.

[3] Kirkton RD, Santiago-Maysonet M, Lawson JH, et al. Bioengineered human acellular vessels recellularize and evolve into living blood vessels after human implantation. Sci Transl Med. 2019;11(485):eaau6934.

[4] Kirkton RD, Prichard HL, Santiago-Maysonet M, Niklason LE, Lawson JH, Dahl SLM. Susceptibility of ePTFE vascular grafts and bioengineered human acellular vessels to infection. J Surg Res. 2018;221:143-51.

[5] Fox CJ, Gillespie DL, O'Donnell SD, et al. Contemporary management of wartime vascular trauma. J Vasc Surg. 2005;41(4):638-44.

[6] White JM, Stannard A, Burkhardt GE, Eastridge BJ, Blackbourne LH, Rasmussen TE. The epidemiology of vascular injury in the wars in Iraq and Afghanistan. Ann Surg. 2011;253(6):1184-9.

[7] Patel JA, White JM, White PW, Rich NM, Rasmussen TE. A contemporary, 7-year analysis of vascular injury from the war in Afghanistan. J Vasc Surg. 2018;68(6):1872-9.

[8] Rasmussen T, Stockinger Z, Antevil J, et al. Wartime vascular injury. Mil Med. 2018;183(suppl_2):101-4.

[9] Vertrees A, Fox CJ, Quan RW, Cox MW, Adams ED, Gillespie DL. The use of prosthetic grafts in complex military vascular trauma: a limb salvage strategy for patients with severely limited autologous conduit. J Trauma. 2009;66(4):980-3.

[10] Greer LT, Patel B, Via KC, Bowman JN, Weber MA, Fox CJ. Management of secondary hemorrhage from early graft failure in military extremity wounds. J Trauma Acute Care Surg. 2012;73(4):818-24.

[11] Humacyte Human Acellular Vessel (HAV) in Patients with Vascular Trauma. ClinicalTrials.gov identifier: NCT03005418. https://clinicaltrials.gov/ct2/show/NCT 03005418 ? term=Humacyte $\&$ draw=1\&rank=1 Updated 4 March 2021. Accessed 8 March 2021. 